\title{
Un « nouveau professionnalisme » des enseignants
} au Royaume-Uni

A new "professionalism" for teachers in the United Kingdom

Una "nueva profesionalidad" de los profesores en Reino Unido

\section{Elaine Wilson}

Traducteur : Charlotte Levrard

\section{Q OpenEdition}

\section{Journals}

Édition électronique

URL : http://journals.openedition.org/ries/951

DOI : $10.4000 /$ ries.951

ISSN : 2261-4265

Éditeur

Centre international d'études pédagogiques

\section{Édition imprimée}

Date de publication : 1 décembre 2010

Pagination : 107-118

ISBN : 978-2-8542-583-1

ISSN : $1254-4590$

Référence électronique 


\section{Un « nouveau professionnalisme » des enseignants au Royaume-Uni ${ }^{\star}$}

\section{Elaine Wilson}

Au Royaume-Uni, des réformes majeures ont vu le jour, depuis le milieu des années soixante-dix, concernant les établissements scolaires et la formation des enseignants. Cette politique rationaliste et technique a eu pour effet de modifier la nature du métier d'enseignant, en introduisant la concurrence du marché et en renforçant la surveillance de l'État. La loi sur la réforme de l'éducation de 1988, votée par le gouvernement conservateur de l'époque, a fusionné habilement forces du marché et contrôle de l'État. Cette tendance s'est développée jusqu'aux années quatre-vingt-dix et, sous les nouveaux travaillistes, s'est développée pour renforcer et pérenniser le changement dans la façon de concevoir le métier d'enseignant. Le Livre blanc de 1998, intitulé Enseignants à la rencontre $d u$ défi $d u$ renouveau, est allé jusqu'à établir la liste des tâches qui incombent à des enseignants modernes, selon les vues du gouvernement, à savoir : "accepter de rendre des comptes»; chercher à "fonder ses décisions sur ce qui a fait ses preuves » dans les établissements scolaires au Royaume-Uni et au plan international ; "travailler en partenariat avec d'autres personnels » dans les établissements scolaires; " accueillir la contribution que peuvent apporter les parents, les entreprises et autres éléments extérieurs à l'établissement ».

Cette intervention gouvernementale dans les établissements se retrouve dans l'approche adoptée en matière de formation des enseignants. Dans les années soixante-dix, l'enseignement est devenu un métier réservé aux diplômés de l'université et la formation des enseignants, en s'éloignant des anciens instituts de formation des enseignants a été intégrée au système général d'enseignement supérieur. Depuis peu, la plupart des futurs enseignants suivent une formation d'un an (PGCE : Post Graduate Certificate of Education) après avoir obtenu une licence dans une discipline donnée. Une petite minorité prépare un diplôme universitaire d'enseignement en trois ou quatre ans, combinant une formation d'enseignant et la préparation d'un diplôme dans une discipline. Depuis les années quatre-vingt-dix, environ $10 \%$ des enseignants obtiennent leur diplôme d'enseignant qualifié (QTS : Qualified teaching status), en suivant une formation en milieu scolaire, sans forcément obtenir un diplôme d'enseignement supérieur.

* Article traduit par Charlotte Levrard. 
De plus, en Angleterre, la formation se déroule désormais très largement en milieu scolaire, y compris dans les programmes proposés par des universités. Le discours actuel privilégie la formation pratique par rapport aux études, ainsi que l'acquisition de compétences pratiques définies par le gouvernement au niveau central (Furlong, 2000).

En 1984, la gamme très diversifiée de programmes de formation d'enseignants offerts par des universités anglaises s'est vue soumise à des obligations définies par le gouvernement qui n'accordait l'accréditation que si ces critères officiels étaient satisfaits. Au cours des années quatre-vingt, ce contrôle centralisé s'est renforcé avec la publication d'une série de circulaires gouvernementales définissant les compétences requises pour l'obtention d'un diplôme d'enseignant qualifié.

À la fin des années quatre-vingt-dix, ces compétences se sont transformées en 85 pages de "programme national (national curriculum) pour la formation des enseignants, qui s'est révélé par la suite irréalisable. On a donc abandonné les compétences au profit d'une définition de critères professionnels (standards) que tous les stagiaires devaient remplir, mais sans méconnaître pour autant l'importance de la réflexivité sur les pratiques. La définition la plus récente de ces critères fournit un cadre couvrant toute la carrière de l'enseignant et non ses seuls débuts (TDA, 2010). Ils se caractérisent comme suit : statut d'enseignant qualifié $(\mathrm{Q})$; critères de base pour les enseignants relevant du barème commun ayant réussi un stage pédagogique d'un an à la suite de leur formation initiale (C) ; enseignants expérimentés ayant atteint le dernier échelon du barème commun $(\mathrm{P})$; enseignants excellents $(\mathrm{E})$ et enseignants « hors classe " (AST : advanced skills teachers). Les critères sont désormais définis par l'Agence pour le développement de la profession enseignante (TDA : Teacher Development Agency), un organisme public non gouvernemental mis en place pour améliorer le niveau scolaire. Ces standards s'organisent autour de trois ensembles d'éléments reliés entre eux : compétences professionnelles transversales; connaissances professionnelles et compréhension du métier; compétences professionnelles. Ils visent à définir clairement ce qui est requis à chaque étape de la carrière. Chaque groupe de critères est construit à partir du niveau précédent, si bien que l'enseignant évalué pour atteindre le dernier échelon doit satisfaire aux exigences du niveau $(\mathrm{P})$ après avoir atteint le niveau $(\mathrm{C})$; l'enseignant qui aspire au titre d'enseignant excellent doit satisfaire aux critères spécifiques de ce statut $(E)$, ainsi qu'à ceux des niveaux précédents $(\mathrm{C}$ et $\mathrm{P})$; et l'enseignant qui souhaite accéder à la hors classe doit remplir les critères correspondant à ce nouveau niveau (A) ainsi qu'à tous ceux des niveaux (C, P et E), (TDA, 2010).

Actuellement, il existe plusieurs parcours de formation pour devenir enseignant :

- une préparation au métier d'enseignant avant la licence en même temps qu'une formation pour un diplôme de premier cycle dans une discipline ; 
- une formation d'enseignant destinée aux diplômés (PGCE) de l'université, dispensée dans des établissements d'enseignement supérieur ainsi que dans le cadre de programmes de formation initiale d'enseignants centrés sur la pratique en milieu scolaire (SCITT), conçus et proposés par des groupements locaux d'établissements scolaires ;

- des parcours de formation d'enseignants basés sur l'emploi, où des enseignants sont payés comme enseignants non qualifiés tout en suivant une formation qualifiante et en travaillant à plein temps dans un établissement scolaire (TDA, 2010).

La formation à l'enseignement dans le second degré concerne actuellement presque exclusivement les diplômés de l'université. C'est ainsi que le pourcentage des cours proposés au niveau du premier cycle est tombé de $14 \%$ à $5 \%$. Le tableau suivant montre, pour 2009, les chiffres pour les différents parcours au stade de l'enseignement primaire ou secondaire.

\begin{tabular}{|l|c|c|}
\hline & Écoles primaires & Établissements du secondaire \\
\hline Formation PGCE & 14172 & 14293 \\
\hline Formation SCITT & 761 & 779 \\
\hline Formation basée sur l'emploi & 2387 & 4454 \\
\hline
\end{tabular}

\section{QUI SUIT UNE FORMATION D'ENSEIGNANT ?}

Le nombre de nouveaux enseignants recrutés pour suivre une formation d'enseignant se calcule chaque année en fonction des besoins globaux en nombre et en type d'enseignants, ainsi qu'en fonction des prévisions de départs à la retraite. Environ $10 \%$ du corps professoral quitte le métier chaque année, dont la proportion la plus élevée provient des établissements primaires. Une proportion plus élevée de femmes quitte à la fois les établissements du primaire et du secondaire (Smithers \& Robinson, 2009).

$\mathrm{Au}$ cours des dix dernières années, on n'a pas constaté une grande variation dans le pourcentage d'hommes diplômés qui suivent des formations PGCE destinées aux futurs instituteurs et dispensées dans les universités ou dans le cadre des formations SCITT. Le pourcentage des minorités ethniques a connu une augmentation qui ne dépasse cependant pas $8 \%$. La proportion de débutants âgés de 25 ans n'a presque pas changé depuis 1999-2000. Dans le secteur du primaire, les demandes dépassent le nombre de places disponibles, les fournisseurs (c'est ainsi que l'on désigne désormais les universités et les établissements scolaires) pouvant opérer une sélection basée sur le mérite. En conséquence, l'on a vu augmenter de 11 \%, depuis 1999, le nombre d'étudiants aux diplômes reconnus qui se destinent à l'enseignement dans le primaire. 
Pour l'enseignement dans le secondaire, la proportion d'hommes recrutés a baissé un peu dans les années quatre-vingt pour se stabiliser aujourd'hui autour de $37 \%$. Le pourcentage issu de minorités ethniques a doublé jusqu'à atteindre $15 \%$. La proportion de nouvelles recrues âgées de 25 ans et plus est restée autour de $56 \%$.

Caractéristiques des nouveaux enseignants recrutés en 2009

\begin{tabular}{|l|c|c|}
\hline & Écoles primaires & $\begin{array}{c}\text { Établissements } \\
\text { du secondaire }\end{array}$ \\
\hline Pourcentage d'hommes & 14 & 37 \\
\hline Pourcentage de monorités ethniques & 8 & 56 \\
\hline Pourcentage de 25 ans et plus & 38 & 95 \\
\hline Pourcentage de diplômés niveau master & 57 & 53 \\
\hline Pourcentage de diplômés niveau licence & 60 & 56 \\
\hline
\end{tabular}

Source : Smithers et Robinson, 2009.

\section{INCITATIONS FINANCIÈRES}

Les enseignants exerçant dans les établissements publics doivent avoir un statut d'enseignant qualifié et leur progression dans le barème se calcule selon l'ancienneté et le degré de compétence atteint. Or il demeure possible d'occuper un poste d'enseignant dans un établissement privé payant sans aucune formation pédagogique. Néanmoins ces établissements se sont évertués, ces dernières années, à former tous leurs enseignants en vue de l'obtention du statut d'enseignant qualifié (QTS).

Dans le secteur public, après obtention du QTS, des enseignants débutants en milieu scolaire commencent normalement au premier échelon du barème, quoiqu'il arrive que de nouveaux enseignants ayant d'autres expériences pédagogiques débutent à un échelon supérieur. Les établissements sont autorisés à augmenter à leur discrétion le nombre de points en fonction d'autres expériences jugées pertinentes. Chaque établissement a sa propre politique salariale qui définit les critères pour la distribution de ces points. L'augmentation des salaires des enseignants se fait selon la révision des barèmes et des indemnités. Au début de chaque année scolaire, en septembre, les enseignants avancent à l'échelon suivant du barème si leurs résultats sont jugés satisfaisants; dans certains cas, ils peuvent avancer de deux échelons si leurs résultats sont jugés excellents. Les enseignants qualifiés qui arrivent au dernier échelon du barème commun peuvent demander une évaluation qui sera effectuée selon huit critères nationaux. Si l'évaluation est positive, on considère qu'ils ont franchi le «seuil » pour passer au barème hors classe. Ce seuil permet aux bons enseignants de progresser de l'échelon 6 à une fourchette de salaire plus élevée. 
Salaire annuel des enseignants 2010

\begin{tabular}{|l|c|c|c|c|}
\hline Échelons & $\begin{array}{c}\text { Angleterre } \\
\text { et Pays de Galles } \\
\text { sauf Londres }\end{array}$ & Ville de Londres & $\begin{array}{c}\text { Proche banlieue } \\
\text { de Londres }\end{array}$ & Région de Londres \\
\hline & $\mathfrak{E}$ & $\mathfrak{E}$ & $\mathfrak{£}$ \\
\hline 1 & 21588 & 27000 & 25117 & 22626 \\
\hline 2 & 23295 & 28408 & 26674 & 24331 \\
\hline 3 & 25168 & 29889 & 28325 & 26203 \\
\hline 4 & 27104 & 31446 & 30080 & 28146 \\
\hline 5 & 29240 & 33865 & 32630 & 30278 \\
\hline 6 & 31552 & 36387 & 35116 & 32588 \\
\hline
\end{tabular}

Source : School Teacher's Review Body.

\section{FORMATION INITIALE ET STAGES PRATIQUES \\ Contrôle qualité dans la formation pédagogique initiale}

Depuis la fin des années quatre-vingt-dix, les cours universitaires préparant au certificat post-universitaire d'aptitude professionnelle à l'enseignement (PGCE) sont soumis au contrôle d'un organisme extérieur d'inspection, l'Ofsted (Office for Standards in Education). Les rapports et les palmarès de résultats sont consultables par le grand public et par les étudiants éventuels sur le site Internet de l'Ofsted (Ofsted, 2010). Les inspections régulières des programmes de formation initiale visent :

- l'établissement d'un rapport sur les résultats des stagiaires, la qualité globale de la formation, de sa direction et de sa gestion, ainsi que la vérification de sa qualité ;

- l'identification des forces et des faiblesses et la mise en lumière des pratiques positives, afin d'améliorer les résultats des stagiaires et donc de leurs formateurs ;

- la valorisation du respect de la réglementation;

- l'encouragement parmi les "organismes fournisseurs de formation" d'une culture d'auto-évaluation rigoureuse, visant à de constantes améliorations et au maintien d'une formation de très grande qualité.

C'est la première fois que le cycle d'inspections 2008-2011 propose un cadre unique pour l'inspection, qui couvre tous les parcours de formation aboutissant à l'obtention du statut d'enseignant qualifié (QTS). Le processus 
d'inspection est décidé selon les résultats du rapport d'inspection précédent. Chaque université ou autre fournisseur produit un document d'auto-évaluation détaillé (SED : self evaluation document), sur la base duquel est établi le déroulement du processus d'inspection. Si l'Ofsted a déjà évalué des programmes universitaires ou autres comme "bien» ou exceptionnels, il leur applique un autre barème pour l'inspection que les cours jugés satisfaisants ou inadéquats. Le processus d'inspection privilégie deux critères principaux d'évaluation: «l'efficacité globale de la formation en vue d'obtenir des résultats de grande qualité chez les stagiaires, et une capacité de pilotage et de gestion à tous les niveaux pour garantir les progrès et/ou maintenir des résultats de grande qualité. »(Cadre Ofsted, 2008).

Le financement et le nombre d'étudiants sont directement liés aux résultats des inspections.

\section{Contrôle des salaires et objectifs de développement dans les établissements scolaires}

S'ajoutant aux inspections aussi régulières que rigoureuses, la performance de l'enseignant est également évaluée par ses supérieurs immédiats dans un contexte pédagogique. En 2003, "Élever le niveau et reconsidérer la charge de travail : un accord national ${ }^{1}$ " a fait entrer la gestion de la performance dans le contexte scolaire, c'est-à-dire le processus qui permet d'évaluer la performance globale d'un enseignant ou d'un directeur d'établissement dans le cadre de son profil de poste.

Dans les établissements publics, la performance des enseignants et des cadres intermédiaires est désormais soumise tous les ans à une évaluation réalisée par l'équipe de direction (conseil d'administration, direction et responsables de domaine) au sein d'un établissements scolaire donné. Tout établissement public est tenu de montrer le lien entre sa gestion de la performance, l'amélioration de ses résultats et son projet de développement.

La gestion de la performance est liée aux salaires à travers le document définissant les salaires et conditions des enseignants (SCTPCD : School Teachers' Pay and Conditions Document), qui, depuis 2004, a introduit l'obligation légale pour les établissements de mettre en place une politique salariale définissant les bases de calcul du salaire des enseignants. Cependant, pour rendre les choses plus compliquées encore, il existe deux catégories d'établissements publics, à savoir les Academies et les instituts technologiques municipaux, qui sont exemptés des règles du SCTPCD. À l'heure actuelle, les Academies sont fondées grâce à un partenariat entre sponsors du monde des affaires, groupes

1. Élaboré par des représentants du gouvernement, des employeurs et des syndicats enseignants. 
confessionnels ou associatifs et le gouvernement ainsi que les autorités éducatives locales. Les instituts de technologie municipaux sont des établissements publics gratuits, ouverts à tous, offrant aux élèves la possibilité de suivre des programmes conçus avec l'aide de sponsors du secteur privé pour faciliter l'entrée dans le monde du travail.

Chaque année, l'évaluation de la performance de l'enseignant est basée sur une observation de ses méthodes pédagogiques et s'appuie sur d'autres éléments tels que les résultats des élèves aux examens. On évalue l'enseignant par rapport à ces critères, et lorsqu'il remplit les conditions définies dans le SCTPCD, il peut espérer une augmentation de salaire. Les résultats de l'évaluation, liés inextricablement aux projets de développement global de l'établissement, conditionnent ce qui sera exigé de l'enseignant dans le cadre de son développement professionnel.

\section{LA PRISE EN COMPTE Du Processus de Bologne}

Depuis septembre 2006, les programmes universitaires préparant au certificat post-universitaire d'aptitude professionnelle à l'enseignement se sont alignés sur les descriptifs de diplômes du Processus de Bologne. Les établissements d'enseignement supérieur ont déterminé chacun le niveau de certification existant qui permettait de justifier de ce «certificat d'aptitude professionnelle à l'enseignement ", au niveau de la licence avec mention en quatre ans (Honours degree) ou du « certificat post-universitaire d'aptitude professionnelle à l'enseignement » au niveau du master.

Le programme au niveau du master exige des étudiants l'apprentissage et l'utilisation des méthodes de la recherche en éducation au sein de l'établissement scolaire où ils sont affectés pendant leur année de formation initiale, afin d'y mener des projets de recherche à petite échelle.

Il faut relever qu'en dépit de ce déplacement au niveau du master universitaire, les compétences exigées des enseignants prennent moins en compte la production et l'utilisation de résultats de recherche. Effectivement, les recommandations actuelles pour 2008-2011, contrairement à celles de 2002, ne font pas spécifiquement mention d'une utilisation de la recherche.

Cette contradiction a été aggravée par la publication en 2007 du Projet pour construire un futur plus prometteur pour nos enfants ${ }^{2}$, qui stipule : "Nous voulons que l'enseignement devienne un métier de niveau master, afin de contribuer à la réalisation de nos ambitions pour tous les enfants et d'améliorer encore le statut de l'enseignant... Notre nouvel objectif est l'obtention d'un master par tous les enseignants au cours de leur carrière. »(DCSF, 2007:4.24).

2. Children's Plan: Building Brighter Futures. 
Cette publication a mené à l'introduction en 2010, dans certains établissements scolaires, d'un projet pilote «Master d'enseignement et d'apprentissage »(MTL : Masters in Teaching and Learning).

\section{DifFICULTÉS ENCORE À RÉSOUDRE \\ Recrutement et maintien dans le métier}

Le recrutement a connu une augmentation au cours de la dernière décennie en dépit des problèmes posés par le recrutement des hommes dans l'enseignement primaire et par l'enseignement des mathématiques et des sciences physiques dans le secondaire. Le recrutement des enseignants est particulièrement délicat au Sud de l'Angleterre et dans les zones urbaines où les établissements scolaires sont perçus comme difficiles. Cependant, si le recrutement a connu une embellie, le maintien des enseignants dans le métier demeure très problématique. En Angleterre, environ $40 \%$ des nouveaux diplômés n'exercent plus après cinq années d'exercice. Il est difficile de déterminer les facteurs d'explication. Il n'en demeure pas moins que les premières données suggèrent qu'à longue échéance, le défi que posent le recrutement et le maintien dans le métier d'un plus grand nombre d'enseignants pourrait se résoudre par une sélection plus sévère, un soutien plus approprié, et une meilleure adéquation entre l'enseignant et l'établissement de première affectation (Ashby et al., 2008).

\section{Défis pédagogiques posés par des établissements difficiles}

En Angleterre, l'éducation dans le secondaire reflète une grande mixité sociale, si bien que trouver des enseignants désireux de rester dans des établissements urbains s'avère encore difficile. Pour traiter ce problème, un Livre blanc parlementaire de 2009 intitulé Nouvelles opportunités (New Opportunities White Paper) a lancé un plan d'incitations comprenant des "menottes d'or ", c'est-àdire $10000 \mathfrak{L}^{3}$ pour encourager les enseignants à rester dans le même établissement difficile pendant trois ans. De plus, les enseignants nouvellement certifiés affectés dans ces établissements se voient offrir la possibilité de participer au projet « Master d'enseignement et d'apprentissage » ainsi que d'accéder au réseau, financé par le gouvernement, des enseignants des établissements qui participent à ce projet. Des enseignants plus expérimentés, qui remplissent les critères professionnels requis, peuvent aussi demander à se voir attribuer les nouveaux postes d'enseignant hors classe ou excellent proposés par les établissements participant au projet.

3. Soit $11221 €(N d I R)$. 


\section{L'importance de la formation des enseignants}

Au Royaume-Uni, les gouvernements successifs ont insisté sur l'importance de la réussite scolaire : pour mesurer cette réussite, ils ont utilisé la performance des élèves aux examens clés, en la mettant en relation avec les résultats des comparaisons internationales. Pour répondre à cette priorité, les établissements se sont concentrés sur la préparation des élèves aux examens importants dont ils relèvent les résultats et les données. Il n'est pas surprenant que l'attention accordée au développement professionnel se soit concentrée sur les initiatives globales prises par l'établissement pour améliorer la performance des élèves aux épreuves. Effectivement, à l'heure où nous écrivons, l'une des conditions requises pour l'obtention d'un diplôme d'enseignant est la capacité de lire et d'interpréter les résultats de performance des établissements.

Des recherches récentes ont mis en question les recommandations de cette approche centralisée de la politique éducative. Wiliam (2010) a constaté que $7 \%$ de la variation entre établissements selon les standards actuels d'évaluation de la performance sont dus à l'influence de l'établissement. Les $93 \%$ restants sont dus à des facteurs que l'établissement ne peut pas contrôler (Wiliam, 2010). De fait, dans un établissement moyen, au Certificat général d'éducation secondaire (GCSE), quinze élèves sur trente d'une même classe auront de bonnes notes dans cinq matières, y compris en anglais et en mathématiques. De plus, si les mêmes élèves se trouvent dans un établissement réputé "bon ", dix-sept sur trente obtiendront ces résultats, alors que seulement treize sur trente auront ces mêmes résultats dans un établissement réputé «mauvais".

Wiliam va plus loin en faisant valoir que l'établissement lui-même a peu d'importance; ce qui compte, c'est la classe dans laquelle l'élève se trouve. D'ailleurs, dans les classes des enseignants les plus efficaces, les élèves issus de milieux défavorisés apprennent autant que ceux issus d'un milieu favorisé et les élèves aux problèmes de comportement apprennent autant que ceux qui n'en ont pas.

Wiliam en conclut sans ambages que ce qui importe vraiment est « la qualité de l'enseignant»(Wiliam, 2009). Barber et Mousha (2007) arrivent à la même conclusion dans leur rapport. Sans être neuve, cette idée s'est cependant égarée dans la pléthore d'initiatives appliquées globalement de haut en bas à tout les établissements scolaires qui domine actuellement la politique éducative au Royaume-Uni. Ces conclusions éclairent cependant l'importance d'une bonne formation des enseignants à toutes les étapes de leur carrière. 


\section{Renouveau de la professionnalisation dans la formation des enseignants}

Les départements d'éducation des universités se sont trouvés confrontés à des défis très importants résultant d'une politique technique et rationaliste visant principalement à établir « ce qui a fait ses preuves » en matière de formation initiale des enseignants.

Le « nouveau professionnalisme » est devenu un outil de contrôle extrêmement puissant, où le respect des recommandations des programmes nationaux et des exigences de l'inspection conditionne directement le nombre de places pour les étudiants et, par voie de conséquence, le financement crucial. Ce financement dépend en outre de l'établissement de parcours de formation non universitaires, basés en grande partie dans des établissements scolaires. De plus, la publication de rapports de l'inspection a abouti à un climat de concurrence entre les différents départements universitaires et les établissements scolaires proposant des programmes de formation. Cela a pour effet global de miner l'efficacité de la planification au niveau régional et de bloquer l'apprentissage réciproque et l'innovation.

Un autre résultat significatif de cette politique technique et rationaliste est la marginalisation grandissante de l'enseignement supérieur dans la formation initiale des enseignants, et dans la formation continue en particulier. Le vocabulaire employé dans les textes nationaux montre combien la formation des enseignants est dominée par la référence aux notions de "critères, compétences, efficacité, excellence ", aboutissant ainsi à un rétrécissement progressif des formes de connaissances professionnelles offertes au métier d'enseignant (Furlong, 2008).

De surcroît, au sein des universités, l'ambivalence à l'égard des formateurs d'enseignants entraîne d'autres conséquences : en Angleterre et au Pays de Galles, au début des années quatre-vingt-dix, s'est développée une tendance au recrutement d'enseignants hautement qualifiés ayant une longue pratique pédagogique, pour enseigner à l'université. Ces enseignants ayant exercé en milieu scolaire avant de se trouver dans le supérieur visaient un changement de carrière. Ces dernières années, le secteur universitaire s'efforce de marginaliser ces formateurs d'enseignants, orientés davantage vers la pratique que leurs collègues universitaires, sous prétexte que leur résultats de recherche sont plus faibles. Cela est dû aux pressions de plus en plus fortes pour conditionner le financement des universités à leurs activités de recherche. Cependant, ce sont précisément ces personnels qui sont déterminants pour la formation professionnelle des enseignants; ce sont eux qui ont une expérience récente de la pratique devant les élèves, ce qui leur permet de faire le lien entre la pratique et d'autres formes de connaissances universitaires codifiées. 
Pour conclure, la centralisation de la production de connaissances théoriques dans le domaine de l'éducation et l'idée d'un "modèle unique " d'enseignement et d'enseignant, s'ajoutant à une régulation externe à tous les niveaux, contribuent à l'amenuisement de l'identité professionnelle des enseignants et de leurs formateurs. Cette identité qui se caractérisait par une "vocation personnelle » se voit amoindrie par un accroissement de la concurrence et l'omniprésence d'une culture de l'évaluation dont le résultat global est une dévaluation du métier d'enseignant.

\section{BiBLIOGRAPHIE}

ASHBY P., HOBSON A., TRACEY L., MALDEREZ A., TOMLINSON P., ROPER P., CHAMBERS G. and HEALY J. (2008) : Beginner Teachers' Experiences of Initial Teacher Preparation, Induction and Early Professional Development: A review of literature. London; Department for schools children and families.

BARBER M. \& MOUSHAD B. (2007): How the best school system come out on top. McKinsey and Company.

FURLONG J. (2000) : Higher Education and the New Professionalism for Teachers: Realising the potential of partnership. A discussion paper (SCOP/CVCP).

FURLONG J. (2008) : 'Making teaching a 21st century profession: Tony Blair's big prize', Oxford Review of Education, 34: 6, 727-739.

Ofsted (consulté le $1^{\text {er }}$ septembre 2010). http://www.ofsted.gov.uk/.

SMITHERS A. \& ROBINSON P. (2009): The Good Teacher Training Guide 2009. Buckingham: CEER.

Training and Development Agency for Schools (TDA) (2008) : Professional Standards for Qualified Teacher Status and Requirements for Initial Teacher Training (revised 2008) (London, TDA). http://www.tda.gov.uk/upload/resources/pdf/p/professional_ standards_2008.pdf (consulté le 1 ${ }^{\text {er }}$ septembre 2010).

Training and Development Agency for Schools (2009) : The Masters in Teaching and Learning (MTL) (London, TDA). http://www.tda.gov.uk/upload/resources/pdf/m/ mtl_faqs.pdf (consulté le 18 octobre 2009).

Training and Development Agency for Schools (TDA) (2008) : What are the professional standards? http://www.tda.gov.uk/teachers/professionalstandards/standards.aspx (consulté le $1^{\text {er }}$ septembre 2010).

WILIAM D. (2010) : Standardized testing and school accountability. Educational Psychologist, 45 (2), 107-122. 
